$$
\begin{array}{lllllllll}
\text { A } & \text { R } & \text { T } & \text { I } & \text { C } & \text { U } & \text { L } & 0 & \text { S }
\end{array}
$$





\title{
Competencias y formación universitaria del documentalista* en el Espacio Europeo de Educación Superior (EEES): estudio de su adecuación al entorno laboral
}

\author{
Carlos Oliva Marañón **
}

Artículo recibido:

18 de enero de 2012.

Artículo aceptado:

23 de julio de 2012.

\section{RESUMEN}

Las competencias del documentalista se han transformado con la implantación del Grado en Información y Documentación adaptado al Espacio Europeo de Educación Superior (EEES). Los objetivos de esta investigación son analizar tanto las ofertas de empleo existentes para el área de Biblioteconomía y Documentación en el ámbito público y en la empresa privada, como los planes de estudios del Grado en Información y Documentación de la Universidad Complutense de Madrid y de la Universidad de Sheffield (Reino Unido) para verificar si se adecuan a las demandas de los empleadores. Se ha consultado la lista de distribución Infodoc, el Diario Oficial de la Unión Europea y las páginas Web

* Cabe recordar que cuando en España se habla de documentalista en México nosotros decimos bibliotecólogo. (N. del C.)

** Universidad Rey Juan Carlos, España. carlos.oliva.maranon@urjc.es

INVESTIGACIÓN BIBLIOTECOLÓGICA, Vol. 26, Núm. 58, septiembre/diciembre, 2012, México, ISSN: 0187-358X. pp. 15-45 
de las Universidades citadas. Se confirma tanto la escasa adecuación entre los contenidos académicos de la titulación y las demandas de empleo en el ámbito público, como un desconocimiento de la titulación por parte de los empresarios. Se aportan algunas propuestas para mejorar la titulación de Grado en Información y Documentación con el objetivo de que los titulados se incorporen al mercado laboral con las máximas garantías.

Palabras clave: Documentalista; Formación; Competencias; Grado en Información y Documentación; Espacio Europeo de Educación Superior (EEES); Mercado laboral; España; Reino Unido.

\section{ABSTRACT \\ Competences and University training of Documentalists in the European Higher Education Area (EHEA): study of adequacy to the job environment Carlos Oliva Marañón}

The competences of the Documentalist have undergone change with the introduction and adaptation of the Degree in Information and Documentation to the European Higher Education Area (EHEA). The aims of this research are to analyze the existing Librarianship and Documentation employment market in both the public and private sectors, and to assess the course of studies Degree in Information and Documentation offered by the Universidad Complutense de Madrid and Sheffield University meet the needs of employers. This research reviews the distribution list of Infodoc, the Official Diary of the European Union and the Web pages of the aforementioned Universities, confirming general inadequacy between the academic contents of these degree programs and the job requirement of the public sector. Moreover, employers appear to be completely unaware of these degree programs. Some suggestions for improvement to these courses of study are provided, aimed at better preparing students in accord with demands of the job market.

Keywords: Documentalist; Formation; Competences; Degree in Information and Documentation; European Higher Education Area (EHEA); labor market; Spain; United Kingdom. 


\section{INTRODUCCIÓN Y ESTADO DE LA CUESTIÓN}

— 1 Grado en Información y Documentación, adaptado al Plan Bolonia y Eenmarcado en el Espacio Europeo de Educación Superior (EEES), tiene como objetivo seleccionar, gestionar, organizar y preservar la información y la documentación para que éstas puedan ser utilizadas por terceros, independientemente del lugar donde estén depositadas, o de sus formatos y soportes. Todo ello teniendo en cuenta muy especialmente el entorno digital que conforma la sociedad actual. Las nuevas tecnologías y especialmente Internet se han introducido ya en las unidades de información. Las bibliotecas virtuales se están convirtiendo en la consecuencia final de la inserción de los nuevos hábitos de trabajo en la cadena documental.

El estudiante del Grado en Información y Documentación tendrá que poner en práctica un conjunto de actitudes y aptitudes no sólo durante la etapa de formación universitaria, sino también en su futuro ejercicio profesional, éstas son: implicación en el aprendizaje (y en el aprendizaje para toda la vida); curiosidad intelectual; capacidad de trabajo en equipo; sentido de la responsabilidad; comprensión lectora, y actitud crítica.

En cuanto a la inserción laboral de los diplomados y licenciados en el área de Biblioteconomía y Documentación se pueden mencionar, entre otros, el estudio de Montes López (1995), quien efectuó el análisis de una muestra de 98 ofertas de trabajo para documentalistas, archiveros y gestores de información que fueron publicadas en los periódicos $A B C$ y El País durante el periodo comprendido entre 1984 y 1994. Los resultados indican que más de la mitad de las ofertas de empleo correspondían a puestos de documentalistas en empresas privadas. Entre los requerimientos se pedía mayoritariamente titulación académica de grado superior y estudios de formación complementaria en Biblioteconomía y Documentación.

Por su parte Alonso y Vázquez (2000) efectuaron un análisis de las ofertas de empleo publicadas a través de la lista de correo electrónico InfoDOC. Los resultados muestran que durante el bienio 1998-1999 se presentaron 317 convocatorias con un total de 855 plazas. Mayoritariamente las ofertas provenían del sector público: bibliotecas (55\% de las plazas), servicios de documentación $(21 \%)$ y archivos (13\%). Las instituciones que más empleo ofertaron fueron las Universidades (217 plazas), seguidas de Ayuntamientos (188) y Ministerios (154). De las 855 ofertas, sólo 59 correspondían a trabajos en empresas privadas.

Un estudio similar realizaron Frías y otros autores (2000), sobre la inserción laboral de los Diplomados en Biblioteconomía y Documentación y los Licenciados en Documentación de la Universidad de Salamanca. En el trabajo 
se analizaron las ofertas de empleo publicadas en el Boletín Oficial de Castilla y León durante el periodo 1991-1998. Los resultados indicaron que la mayoría de las ofertas de empleo correspondían a bibliotecas ( $80 \%$ de las plazas) y un porcentaje menor de las solicitudes $(11 \%)$ era para archivos. La mayor parte de las ofertas laborales (85\%) provenía de la Administración Pública (Ayuntamientos, Diputaciones y Junta de Castilla y León). Por su parte, las Universidades ofrecían un 13\% de las vacantes. En el estudio se comprobó que los titulados trabajaban, principalmente, en bibliotecas públicas (26\%), bibliotecas universitarias (21\%), archivos $(17 \%)$, en instituciones documentales de la Administración Pública (10,5\%) y en empresas privadas (8,4\%).

Moreiro (2001a) estudió también la inserción laboral de los estudiantes y graduados recientes de la Universidad Carlos III de Madrid. En su trabajo, el autor analizó los perfiles requeridos por los empleadores que acudieron a la Bolsa de Empleo del Servicio de Orientación y Planificación Profesional de la Universidad Carlos III. También analizó los informes de satisfacción presentados una vez ocupados los puestos de trabajo y encuestó a los alumnos de la promoción del año 1999.

Los resultados indicaron que durante 1998 se ofrecieron 281 puestos de trabajo (64 de empleo directo y 217 de prácticas profesionales). La mayor oferta fue para los Diplomados (185 puestos), y en menor proporción para los Licenciados (96). El estudio constató también como resultado que las empresas e instituciones empleadoras procedían mayoritariamente de la consultoría (39 empresas consultoras ofertaron plazas para Diplomados y 28 para Licenciados), y de los servicios a empresas ( 37 ofrecieron plazas para Diplomados y 12 para Licenciados). Con respecto a los Diplomados, la mayoría de las ofertas de empleo provenían de la consultoría y de los servicios para empresas, seguida de las artes gráficas (28 empresas) y de la Administración Pública (24 ofertas). Por su parte, en el caso de la Licenciatura, las ofertas de empleo correspondían a la consultoría y los servicios a empresas, seguidos de la Administración Pública (12 solicitudes).

Moreiro (2001b) analizó también la inserción laboral de los alumnos matriculados en la Licenciatura en Documentación de la Universidad Carlos III. Los resultados indicaron que el $72 \%$ de los alumnos del último año estudiaban y trabajaban. La mayor parte del alumnado tenía empleo antes de iniciar la Licenciatura, al igual que el $21 \%$ de quienes cursaban la Diplomatura. Los estudiantes trabajaban en su mayoría en centros y servicios de documentación $(40,5 \%)$, bibliotecas $(32,4 \%)$ y, en menor proporción, en bases de datos documentales $(11,4 \%)$.

Otro estudio de interés fue el efectuado por el Consejo Social de la Universidad Complutense (2001). En la investigación se analizaron mediante 
una encuesta las trayectorias laborales de los titulados en Biblioteconomía y Documentación de la Universidad Complutense entre 1992 y 1997. Los resultados señalaron que los dos sectores donde mayoritariamente se consigue el primer empleo es el de Servicios (61\%), y el de la Administración Pública $(35 \%)$.

Comalat, Espelt y Rubió (2003) estudiaron los convenios firmados por la Facultat de Biblioteconomia i Documentació de la Universitat de Barcelona con empresas privadas. Los convenios analizados fueron sobre prácticas laborales no vinculadas al plan de estudios. Las ofertas que presentaban estas características conformaban el $40 \%$ del total de los convenios ofrecidos a la Facultad por un total de 109 empresas. Éstas se agrupaban en dos grandes sectores de actividad: consultorías empresariales, financieras, legales y de recursos humanos (26) y empresas de contenidos, como prensa y servicios editoriales (21). En el periodo comprendido entre 1998 y 2002, la Facultad firmó 695 convenios (293 con entidades públicas y 402 con empresas privadas). La mayor parte correspondió a estudiantes de primer ciclo (602 convenios).

Otro estudio importante fue el realizado por la Universidad de Barcelona (2003), sobre la inserción laboral de los graduados en Biblioteconomía y Documentación entre 1998 y 2003. La investigación comprobó que las bibliotecas, principalmente las públicas, constituían la principal fuente de empleo para los titulados. Los resultados indicaron que el $24,7 \%$ de los egresados trabajaba en estas unidades de información; un 16\% en bibliotecas universitarias; un 12,4\% en bibliotecas especializadas o centros de documentación de empresas e instituciones; un 10,3\% en bibliotecas especializadas de la Administración Pública; un 7,2\% en archivos; un 3,1\% en bibliotecas de centros de enseñanza, y un 3,1\% en medios de comunicación. Por otra parte en el trabajo se observó que al realizar el estudio, un 33,1\% de los egresados tenía un contrato de trabajo permanente, un $29,4 \%$ un contrato laboral temporal y un $17,5 \%$ eran funcionarios.

Por su parte el análisis de la inserción de los Licenciados presentó resultados similares. Del total de encuestados el 30,3\% trabajaba en bibliotecas públicas; el 21,2\% en bibliotecas especializadas y centros de documentación de la Administración Pública; el 15,2\% en bibliotecas universitarias; el $6,1 \%$ en bibliotecas especializadas y centros de documentación de empresas e instituciones y un 6,1\% en archivos. Con respecto al nivel de los empleos el $47 \%$ correspondía a funcionarios, un $23,5 \%$ a laborales permanentes y un $12 \%$ a laborales temporales.

Tejada Artigas (2007) investigó la situación laboral y profesional en el sector de la Información y la Documentación mediante la comparación de dos encuestas de los años 2001 y 2006, realizadas entre los socios de la de 
la Sociedad Española de Documentación e Información Científica (SEDIC). Las conclusiones principales fueron el alto grado de satisfacción en el empleo $(72,7 \%)$ y la estabilidad laboral, ya que el $69,4 \%$ de la muestra era funcionario o tenía un contrato indefinido.

También los profesores Ubieto, García Marco y Domingo del Valle (2007) investigaron la evolución de la oferta de empleo público en esta área desde 1996 hasta 2006, a través del análisis de los Boletines Oficiales de diferentes Comunidades Autónomas. Concluyeron que el Ministerio de Cultura, con 256 ofertas, seguido de las Universidades, con 182, eran los Organismos que más puestos de trabajo ofertaban. En el ámbito autonómico, la Administración Local ofrecía 73 plazas y las Universidades de cada Comunidad, 54.

Igualmente, Muñoz Cañavate, Chaín Navarro y Salido Martínez (2007) demostraron cómo el ámbito de la gestión de la información en la Administración electrónica representa un nuevo contexto laboral. Además concluyeron que los Licenciados en Documentación pueden realizar trabajos en la Administración Pública centrados, por ejemplo, en el diseño de websites, carga y mantenimiento y actualización de contenidos e interacción con los usuarios. Además, Ríos Hilario y Sánchez Santos (2011) han investigado el perfil de los catalogadores y su adecuación entre la formación universitaria y la demanda laboral.

En America Latina la temática de la inserción laboral ha sido escasamente abordada. Pineda (2002) investigó las características que presentan los empleos a los que acceden los titulados de la Escuela de Bibliotecología de la Universidad de Córdoba (Argentina). Las principales conclusiones constatan el hecho de que las bibliotecas son el principal yacimiento de empleo; que es alto el nivel ocupacional de los titulados, y que la pertinencia de su formación profesional es acorde con las exigencias del mercado laboral.

Azevedo y Gómez (2006) estudiaron la demanda de profesionales de la información mediante el análisis de los anuncios de empleo publicados en los Departamentos de Recursos Humanos de las empresas brasileñas. Concluyeron que estos trabajadores cuentan con las habilidades y requisitos que exige actualmente el mercado laboral en Brasil; y que las principales habilidades exigidas fueron el dominio de las Tecnologías de la Información y la Comunicación (TIC), la Administración y el conocimiento de idiomas.

Artaza (2011) estudió las demandas de empleo mediante el análisis de las ofertas de trabajo en Argentina a través de las listas de distribución Abdam (Lista de interés de la Asociación de Bibliotecarios, Documentalistas, Archiveros y Museólogos de Mar del Plata) y Abgra (Lista de correo electrónico de la Asociación de Bibliotecarios Graduados de la República Argentina). Sus resultados confirmaron una mayor demanda de bibliotecarios frente a los Licenciados 
porque es mayor el número de graduados bibliotecarios solicitados en su mayoría para las bibliotecas escolares. Igualmente existe una mayor demanda de conocimientos vinculados con las áreas de Tecnología y Organización de la Información e idiomas. Además se ha podido constatar que en ambas listas se ofertó empleo por parte de las Bibliotecas y los Centros de Documentación en detrimento de las empresas.

Por tanto esta investigación se centra, por un lado, en el análisis de las ofertas de empleo de Biblioteconomía y Documentación a través de la lista de distribución Infodoc y, por otro, en la comparación de los planes de estudios del Grado en Información y Documentación de las Universidades Complutense de Madrid y de Sheffield (Reino Unido) para adecuarlos a la demanda laboral.

\section{Objetivos}

- Verificar si las demandas de los empleadores se adecuan a la formación académica que reciben los alumnos del área de conocimiento de Información y Documentación.

- Analizar las ofertas de empleo existentes para el área de Biblioteconomía y Documentación, tanto en el ámbito público como en la empresa privada.

- Describir los planes de estudios del Grado en Información y Documentación de la Universidad Complutense de Madrid y de la Universidad de Sheffield (Reino Unido).

- Plantear, razonadamente, las mejoras que tengan que realizarse en dichos planes de estudios para que se adecuen a las ofertas de empleo analizadas, con el objetivo de que los titulados se incorporen al mercado laboral con las máximas garantías.

\section{Metodología}

La metodología que se ha seguido para realizar esta investigación ha sido cualitativa, y de análisis de las ofertas de empleo (ámbito nacional, europeo y privado) y los planes de estudio, a la vez que se han realizado propuestas de mejora para fomentar el éxito laboral.

Se consultó la lista de distribución Infodoc ${ }^{1}$ por su antigüedad, relevancia y pertinencia. Fundada en 1999 y gestionada por la Biblioteca de la Facultad 
de Traducción y Documentación de la Universidad de Salamanca (España), esta lista se ha utilizado para analizar las ofertas de empleo en el área de Biblioteconomía y Documentación, tanto en el ámbito público como en el privado. Esta lista trabaja con información objetiva y su vocación es la distribución de mensajes con información profesional del mundo de las Bibliotecas y la Documentación en general. Su objetivo es que los suscriptores dispongan de una información actualizada en su campo de interés a través del correo electrónico. Está estructurada en tres secciones: novedades, ofertas de empleo y cursos. Además, Infodoc se ha consolidado como un servicio de información, no sólo para los profesionales de la Documentación, sino también para particulares. Por tanto, sus usuarios son investigadores, docentes, profesionales de las bibliotecas y de la información, estudiantes y opositores.

Igualmente, se ha consultado un informe elaborado por la Fundación Banco Bilbao Vizcaya Argentaria (BBVA) (2011), en el que la mayoría de los jóvenes europeos que cursan estudios superiores cree que existe un importante desajuste entre la formación que reciben y la vida laboral.

Por otro lado se han seleccionado las Universidades Complutense de Madrid y de Sheffield por su tradición y prestigio en ofrecer los estudios de Información y Documentación. En concreto, la Facultad de Ciencias de la Documentación de la Universidad Complutense los ofrece desde 1991, heredera de una larga tradición que se remonta a la Escuela Superior de Diplomática, desaparecida en 1900; mientras que el Departamento de Biblioteconomía y Documentación de la Universidad de Sheffield data de 1963. En consecuencia se ha realizado un análisis de los planes de estudios del Grado en Información y Documentación de la Universidad Complutense de Madrid y de la Universidad de Sheffield (Reino Unido), con el fin de verificar cuáles son las deficiencias existentes en los contenidos académicos de esta titulación y proponer mejoras para que los titulados adquieran una mejor formación con vistas a su incorporación al mercado laboral.

\section{LiBRo BLANCO DE LA ANECA Y COMPETENCIAS ESPECÍFICAS DEL GRADO EN INFORMACIÓN Y DOCUMENTACIÓN ${ }^{2}$}

Los Libros Blancos de la Agencia Nacional de Evaluación de la Calidad y la Acreditación (ANECA) recogen aspectos fundamentales para el diseño de un modelo de Título de Grado: análisis de los estudios correspondientes o afines en Europa; características de la titulación europea seleccionada; estudios 
de inserción laboral de los titulados durante el último quinquenio; y perfiles y competencias profesionales, entre otros aspectos. Todo ello adaptado al Espacio Europeo de Educación Superior (EEES) y al Real Decreto 1393/2007 del 29 de octubre, por el que se establece la ordenación de las enseñanzas universitarias oficiales.

En este sentido las competencias específicas del Grado en Información y Documentación son las siguientes:

- Interacción con los productores, usuarios y clientes de la información.

- Conocimiento del entorno profesional de la información y la documentación.

- Conocimiento del marco jurídico y administrativo nacional e internacional de la gestión de la información.

- Identificación, autentificación y evaluación de los recursos de información.

- Gestión de colecciones y fondos.

- Conservación y tratamiento físico de los documentos.

- Análisis y representación de la información.

- Organización y almacenamiento de la información.

- Búsqueda y recuperación de la información.

- Elaboración y difusión de la información.

- Tecnologías de la Información: Informática.

- Tecnologías de la Información: Telecomunicaciones.

- Técnicas de producción y edición.

- Técnicas de gestión administrativa.

- Técnicas de marketing.

- Técnicas comerciales.

- Técnicas de adquisición.

- Técnicas de gestión micro-económica.

- Técnicas de instalación, acondicionamiento y equipamiento.

- Técnicas de planificación y gestión de proyectos.

- Técnicas de diagnóstico y evaluación.

- Técnicas de gestión de recursos humanos.

- Otros conocimientos aplicados a la información y a la documentación.

ANÁlisis DE OFERTAS DE EMPLEO

Se ha considerado pertinente estudiar las ofertas de empleo convocadas en el ámbito público tanto nacional como europeo, así como tres ofertas relevantes de la empresa privada: 
1) Oposiciones de Facultativo de Archivo, Biblioteca o Documentación.

2) Oposiciones o pruebas selectivas para organismos internacionales o europeos.

3) Puestos de trabajo en la empresa privada.

\section{Oposiciones de Facultativo de Archivos, Bibliotecas y Museos ${ }^{3}$}

\section{Universidad Politécnica de Madrid, Facultativos de Archivos, Bibliotecas y Museos, Subgrupo A1.}

Resolución del 15 de junio de 2010 de la Universidad Politécnica de Madrid, por la que se convocan pruebas selectivas para el ingreso en la Escala de Facultativos de Archivos, Bibliotecas y Museos, Subgrupo A1, de la Universidad Politécnica de Madrid, por el sistema de promoción interna y por el sistema de concurso-oposición libre.

\section{Requisitos de los candidatos}

1) Para ser admitido a la realización de las pruebas selectivas, los aspirantes deberán reunir los siguientes requisitos:

a) Tener la nacionalidad española o ser nacional de un Estado miembro de la Unión Europea o nacional de aquellos Estados a los que, en virtud de Tratados Internacionales celebrados por la Comunidad Europea y ratificados por España, sea de aplicación la libre circulación de trabajadores en los términos en que ésta se halle definida en el Tratado Constitutivo de la Comunidad Europea. También podrán participar el cónyuge, ascendientes y descendientes del cónyuge, de los españoles y de los nacionales de otros Estados miembros de la Unión Europea, siempre que no estén separados de derecho, menores de veintiún años o mayores de dicha edad. Igualmente se extenderá a las personas incluidas en el ámbito de aplicación de los Tratados Internacionales celebrados por la Comunidad Europea y ratificados por España, en los que sea aplicable la libre circulación de trabajadores.

b) Tener cumplidos los dieciocho años de edad y no haber alcanzado la edad de jubilación legalmente establecida. 
c) Estar en posesión del título de Licenciado, Ingeniero, Arquitecto o equivalente, o en condiciones de obtenerlo en la fecha en que termine el plazo de presentación de solicitudes. En caso de titulaciones obtenidas en el extranjero deberá estarse en posesión de la credencial que acredite su homologación, o en condiciones de obtenerla.

d) No padecer enfermedad ni estar afectado por limitación física o psíquica que sea incompatible con el desempeño de las correspondientes funciones.

e) No haber sido separado, mediante expediente disciplinario, del servicio de cualquiera de las Administraciones Públicas ni hallarse inhabilitado para el desempeño de las correspondientes funciones. Los aspirantes que posean nacionalidad distinta de la española, además de no encontrarse inhabilitados para el acceso a la Función Pública en España, no deberán estar sometidos a sanción disciplinaria o condena penal que impida, en su Estado, el acceso a la Función Pública.

2) Los aspirantes que concurran a estas plazas por el turno de promoción interna deberán pertenecer como Funcionarios de Carrera a alguno de los Cuerpos o Escalas de los Subgrupos A1 y A2 de la Universidad Politécnica de Madrid, no encontrarse en la situación administrativa de suspensión de funciones, y haber prestado servicios efectivos durante al menos dos años, como Funcionario de Carrera en Cuerpos o Escalas de los Subgrupos A1 y A2 de la Universidad Politécnica de Madrid.

3) Todos los requisitos enumerados en la base ("Para ser admitido...") deberán poseerse en el día de finalización del plazo de presentación de solicitudes y mantenerse hasta el momento de la toma de posesión como Funcionario de Carrera.

\section{Oposiciones de la Unión Europea}

\section{Oficina Europea de Selección de Personal (EPSO) ${ }^{4}$}

Administradores Biblioteconomía -Técnicas de Información- Expertos Audiovisuales

- Núm. plazas: 1

- Administración convocante: Oficina Europea de Selección de Personal (EPSO) 
- Boletín: Diario Oficial de las Comunidades Europeas

- Número de boletín: C 110/A

- Fecha de boletín: 29/04/2010

- Titulación: Ver convocatoria

- Plazo de presentación: Ver convocatoria

- Lugar de presentación: Ver convocatoria

\section{Diario Oficial de la Unión Europea}

EPSO/AD/178/10 - Administradores (AD 5)

Biblioteconomía/Técnicas de Información

1) Naturaleza de las funciones

La función general de los administradores en Biblioteconomía y Técnicas de Información es gestionar y prestar servicios de información profesionales.

Téngase en cuenta que en esta oposición no buscamos especialistas en Tecnología de la Información (TI), archiveros o gestores de documentos.

Entre sus principales tareas, que pueden variar de una institución a otra, figuran las siguientes:

- Gestión de servicios de información, incluida la gestión de servicios de búsqueda de información general y especializada o de una biblioteca, gestión de sitios Intranet de información con bases de datos en línea comerciales y oferta de formación en técnicas de información.

- Gestión de vocabularios controlados, procedimientos de catalogación e indexación; gestión de colecciones; gestión de datos profesionales.

- Búsqueda de información especializada y gestión de recursos de información, especialmente en ámbitos particulares o para grupos de clientes específicos.

- Funciones de dirección profesional o especialización en ámbitos particulares de servicio como referencias, normas técnicas profesionales, formación de clientes, derechos de autor, innovación o evolución de las técnicas de información.

- Gestión de grupos de trabajo y equipos especiales.

- Análisis y resumen de información estratégica, redacción de informes y resúmenes de gestión. 
- Respuesta a las solicitudes de información de los clientes, selección de información, catalogación e indexación de documentos, presentaciones del servicio y formación de clientes.

- Tareas de bibliotecario-jurista (en el Tribunal de Justicia de la Unión Europea en Luxemburgo).

2) Titulación

Un nivel de estudios que corresponda a un ciclo completo de estudios universitarios de tres años como mínimo acreditado por un título en Técnicas de Información, Documentación o Biblioteconomía. (Los estudios en los campos de Tecnologías de la Información y Archivística no están comprendidos en esta definición).

obien:

Un nivel de estudios que corresponda a un ciclo completo de estudios universitarios de tres años como mínimo acreditado por un título, una formación mínima de un año sancionada por un diploma complementado por Técnicas de Información, Documentación o Biblioteconomía. (Los estudios en los campos de Tecnologías de la Información y la Archivística no están comprendidos en esta definición).

\section{Para el perfil de bibliotecario-jurista}

Un nivel de estudios que corresponda a un ciclo completo de estudios universitarios de tres años como mínimo acreditado por un título en Derecho y formación o experiencia profesional de un mínimo de dos años en el campo de la Biblioteconomía.

La descripción del puesto de trabajo se centra en la capacidad de relación personal (clientes, plantilla) y la capacidad de manejo de información en todas sus formas. El interés de estas funciones se centra menos en la gestión tradicional de libros y documentación que en la capacidad de ofrecer información precisa y pertinente que satisfaga las necesidades del cliente. Para lograrlo se requieren muchos tipos de aptitud de gestión en contextos profesionales de manejo de la información: gestión del servicio, comercialización, calidad, gestión de recursos humanos y financieros y gestión de contratos.

Consiguientemente, al evaluar las cualificaciones de los candidatos, el tribunal de selección tendrá en cuenta en especial:

- El conocimiento práctico del proceso y la gestión de los servicios de información. 
- El conocimiento práctico de los servicios a la clientela, la calidad y la comercialización, así como de los servicios de información en línea.

- El conocimiento de las Políticas de la UE y del Derecho Nacional, Internacional y de la UE.

- El conocimiento de las materias siguientes: Ciencias Políticas, Relaciones Internacionales, Economía y Ciencia; o un profundo conocimiento sólido de otros campos pertinentes para la actividad de las instituciones europeas.

- Se valorará positivamente un excelente conocimiento del paisaje informativo y de las lenguas oficiales de uno o más de los países siguientes: Austria, Chipre, Eslovaquia, Estonia, Francia, Irlanda, Letonia, Lituania, Luxemburgo, Malta, Países Bajos, Polonia y Reino Unido.

- Conocimientos prácticos de catalogación e indización.

- Para el perfil de bibliotecario-jurista, el tribunal tendrá en cuenta, especialmente, sin ninguna preferencia lingüística, los conocimientos en Biblioteconomía y los conocimientos en Derecho de la Unión Europea.

\section{Ofertas de empleo privado}

\section{Universidad Europea de Madrid ${ }^{6}$}

Ayudante de Biblioteca para la Biblioteca del Campus Villaviciosa de Odón

Contacto: correo electrónico: recruitment@uem.es

La Universidad Europea de Madrid desea incorporar un Ayudante de Biblioteca para la Biblioteca del Campus de Villaviciosa de Odón.

Gestionará la tramitación y adquisición de todas las peticiones de material bibliográfico. Tendrá interlocución con proveedores y editores relacionados con el servicio, realizará el proceso automatizado de materiales mediante SGB UNICORN (recepción, facturación, catalogación, clasificación, etc.), atenderá demandas de información/documentación de los usuarios y apoyará al resto de las áreas de la Biblioteca.

Información complementaria de la oferta: 
- Requisitos mínimos:

- Estudios mínimos: diplomado en Biblioteconomía y Documentación.

- Experiencia mínima de 2 años en bibliotecas universitarias en el área de adquisiciones y procesamiento de materiales mediante SGB y UNICORN.

- Práctica en la gestión y manejo de proveedores, gestión de presupuestos.

- Nivel alto de Inglés.

- Usuario avanzado de Office (Excel, Word, Internet ...)

- Requisitos deseados:

- Buscamos personas que tengan una clara orientación al cliente, capacidad de trabajo en equipo, buenas habilidades comunicativas, con iniciativa y flexibilidad.

- Se ofrece desarrollo profesional, contrato para vacante estable. Jornada laboral completa, cuyo horario es de lunes a viernes de 9 a $18 \mathrm{~h}$.

Bibliotecario/a y Administrativo/ $\mathrm{a}^{7}$

Lunes, 10 de mayo de 2010, 16:33:38 Madrid (Madrid).

- Empresa: Fundación Japón, Madrid.

- Descripción: Funciones del Puesto: funciones de Bibliotecario/a: se deberán realizar las siguientes actividades en la Biblioteca de la Fundación Japón, Madrid: selección, adquisición, orden y clasificación.

- Requisitos: (1) Nivel de estudios universitarios completados. (2) Alto nivel de los siguientes idiomas: a. Japonés b. Inglés c. Español (en caso de no ser el español la lengua materna) (3) Preferentemente, aunque no imprescindible, la posesión del Título de Biblioteconomía. (4) Permiso de Trabajo indispensable en caso de candidatos/as fuera de la CE. Es imprescindible que el candidato visite la página Web de la Fundación Japón, Madrid para conocer el contenido completo de la oferta y el modo de solicitar el puesto.

- www.fundacionjapon.es 
Indra $b m b^{8}$

Catalogar mapas antiguos

- En Indra bmb estamos buscando un grupo de personas para catalogar mapas antiguos, que tengan experiencia en el uso de UNICORN y el formato MARC21. Sería para un proyecto de 2 años de duración en un organismo público de Madrid.

- Todos los que estén interesados en enviar su CV o tener más información, comunicarse con Francisco Heredia en la siguiente dirección: fheredia@indrabmb.es

\section{DISCUSIÓN DE LAS OFERTAS DE EMPLEO}

En la oferta de empleo público de oposiciones de Facultativo de Archivos, Bibliotecas y Museos convocada por la Universidad Politécnica de Madrid, se exige, entre otros requisitos, poseer el título de Licenciado, Ingeniero, Arquitecto o equivalente, o en condiciones de obtenerlo en la fecha en que termine el plazo de presentación de solicitudes. Se solicita una titulación superior (Licenciado, Ingeniero, Arquitecto o equivalente).

No se adecua al perfil de un titulado superior en Información y Documentación por las siguientes razones:

- Se exige ser Licenciado, por tanto al no especificar una carrera concreta sirven todas las Licenciaturas.

- Se exige ser Ingeniero, por tanto al no especificar una rama concreta sirven todas las Ingenierias.

- Para acceder a la oposición también es válida la titulación de Arquitecto.

- El término equivalente hace referencia a las titulaciones que, oficialmente, estén equiparadas a una Licenciatura, fundamentalmente, las siguientes:

- Estudios cursados en Escuelas Oficiales de Náutica (Real Decreto $1439 / 1975$, de 26 de junio, BOE de 3 de julio, y R.D. 355/1979 de 2 de febrero, BOE de 28 de febrero).

- Enseñanzas Militares de Formación (Ley 17/1999, de 18 de mayo, BOE de 19 de mayo). 
- Título de Técnico de Empresas y Actividades Turísticas (R.D. 865/1980, de 14 de abril, BOE de 9 de mayo).

- Maestros de Primera Enseñanza (Orden de 14 de septiembre de 1978, BOE de 30 de septiembre).

- Título de Graduado Social Diplomado (R.D. 921/1980, de 3 de mayo, BOE de 17 de mayo).

- Determinados títulos en Ciencias Eclesiásticas (R.D. 3/1995, de 13 de enero, BOE de 4 de febrero).

- Título de piloto de transporte de línea aérea (Orden de 9 de mayo de 1995, BOE de 17 de mayo).

- Los siguientes títulos establecidos en la Ley Orgánica 1/1990 (LOGSE) de 3 de octubre, BOE de 4 de octubre y Ley Orgánica 2/2006, de 3 de mayo, de Educación, BOE de 4 de mayo.

- Título de Profesor Superior de Música y sus equivalentes (R.D. 1542/1994, de 8 de julio, BOE de 9 de agosto).

- Título de Conservación y Restauración de Bienes Culturales y sus equivalentes (R.D. 440/1994, de 11 de marzo, BOE de 6 de abril).

- Título Superior de Arte Dramático y sus equivalentes (R.D. 770/1997, de 30 de mayo, BOE de 20 de junio).

- Título Superior de Cerámica.

- Título Superior de Danza.

- Título de Diseño.

- Título Superior de Vidrio.

Por tanto para acceder a dicha oposición tendrían que exigirse únicamente las titulaciones de Graduado en Información y Documentación o Licenciado en Documentación, que son las que se adecuan a las funciones profesionales que se deben desempeñar. Además al existir un número menor de titulados que en otras carreras universitarias hay menos competitividad a la hora de presentarse a la oposición, lo que aumenta las posibilidades de obtener una plaza.

En la oferta de empleo de administradores en Biblioteconomía y Técnicas de Información publicada en el Diario Oficial de la Unión Europea, se pide una titulación media (Diplomado universitario en Biblioteconomía y Técnicas de Información y Documentación), lo que resulta muy adecuado para las funciones que tienen que desempeñar las personas que obtengan plaza.

- Además en la base complementaria se exige cualquier ciclo universitario finalizado de tres años completado con una formación mínima de un año sancionada por un diploma refrendado por Técnicas de Información, 
Documentación o Biblioteconomía. Este requisito indica que se puede presentar cualquier titulado, lo que no resulta adecuado, ya que existe una Diplomatura universitaria específica que prepara para que estos profesionales desempeñen su trabajo con ética y rigor.

- En cuanto al perfil del bibliotecario-jurista, a priori, no es acertada la exigencia de ser titulado en Derecho, ya que para desempeñar este trabajo tendría que ser Graduado en Información y Documentación o Licenciado en Documentación. Además, la formación jurídica necesaria se puede obtener realizando cursos de especialización jurídicos o un Máster Oficial en Derecho de la Unión Europea.

En la oferta de empleo de un Ayudante de Biblioteca para la Universidad Europea de Madrid, el perfil solicitado es muy adecuado por las siguientes razones:

- Específicamente se exige la Diplomatura en Biblioteconomía y Documentación.

- Es necesaria una experiencia laboral previa de dos años en bibliotecas universitarias, concretamente en el área de adquisiciones.

- Exigen manejo de UNICORN y gestión de presupuestos.

- Se solicita un nivel alto de Inglés.

- Igualmente el candidato tiene que ser usuario avanzado de Office.

- Algo muy valorado por las empresa es el trabajo en equipo, las habilidades comunicativas, la iniciativa y la flexibilidad.

En la oferta de empleo de Bibliotecario para la Fundación Japón, el perfil solicitado se considera adecuado en los siguientes términos:

- Se exige el Título de Biblioteconomía (aunque no es imprescindible).

- Se solicita un alto nivel de Inglés y Japonés.

- Se especifican las funciones que hay que realizar.

Sin embargo no se considera pertinente en estos aspectos:

- El título de la oferta (bibliotecario-a / administrativo-a) es inadecuado porque un bibliotecario no es un administrativo y viceversa. Por tanto, tendría que cambiarse y dejarse únicamente en "bibliotecario".

- El Título de Biblioteconomía tendría que ser imprescindible, ya que es la única titulación universitaria que capacita para realizar las funciones descritas. 
- No hace referencia a la posesión de conocimientos de informática.

- Tendría que exigir conocimientos de UNICORN, ABSYS y MARC21.

- Tampoco se solicita experiencia laboral previa en bibliotecas.

- No se especifica que el candidato posea capacidad para trabajar en equipo, ni habilidades comunicativas, aspectos fundamentales en cualquier trabajo.

En la oferta de empleo de catalogador de mapas antiguos para la Fundación Indra, el perfil solicitado es adecuado por las siguientes razones:

- Se exigen conocimientos de UNICORN y MARC21.

No es pertinente en los siguientes aspectos:

- No se solicita titulación específica y por lo tanto sirve cualquiera. Tendría que exigirse el Grado en Información y Documentación, la Licenciatura en Documentación o la Diplomatura en Biblioteconomía y Documentación.

- Tampoco se incluye, como mérito curricular adicional, ser titulado en Geografía y Ordenación del Territorio.

- Igualmente no se exigen conocimientos específicos en Cartografía, Sistemas de Información Geográfica (SIG) como IDRISI, ni en Técnicas de Representación Gráfica.

- No hace referencia a la posesión de conocimientos de informática, en especial los relativos a la utilización de Bases de Datos específicas de Geografía.

- No se exige experiencia profesional en el Instituto Geográfico Nacional (IGN).

DESCRIPCIÓN DE PLANES DE ESTUDIOS

\section{Grado en Información y Documentación (Universidad Complutense) ${ }^{9}$}

Resolución del 28 de mayo de 2010 de la Universidad Complutense de Madrid, por la que se publica el plan de estudios de Graduado en Información y Documentación (BOE núm. 150, de 21 de junio de 2010) (BOCM núm. 191, de 1 de agosto).

9 http://www.ucm.es/?a = estudios \&d=muestragrado $3 \& \mathrm{idgr}=23$ 


\begin{tabular}{|l|c|}
\hline \multicolumn{2}{|c|}{ Tipo de asignatura } \\
\hline Formación Básica & 60 créditos ECTS \\
\hline Obligatorias & 126 \\
\hline Optativas & 42 \\
\hline Prácticas externas & 6 \\
\hline Trabajo de Fin de Grado & 6 \\
\hline Total & 240 \\
\hline
\end{tabular}

\begin{tabular}{|l|c|}
\hline \multicolumn{2}{|c|}{ Primer curso } \\
\hline Historia de España & 6 créditos \\
\hline Lengua Española & 6 \\
\hline Informática General & 6 \\
\hline Teoría de la Comunicación & 6 \\
\hline Instituciones de Derecho Administrativo & 6 \\
\hline Sociología & 6 \\
\hline Industrias culturales & 6 \\
\hline Historia de la Cultura Escrita y de las Bibliotecas & 6 \\
\hline Organización y Gestión de Bibliotecas & 6 \\
\hline Teoría e Historia de la Ciencia de Documentación & 6 \\
\hline
\end{tabular}

\begin{tabular}{|l|c|}
\hline \multicolumn{2}{|c|}{ Segundo curso } \\
\hline Inglés & 6 créditos \\
\hline Géneros Literarios y Transmisión de Textos & 6 \\
\hline Economía de la Información y Documentación & 6 \\
\hline Fuentes Generales de Información & 6 \\
\hline Fuentes de Información Especializada & 6 \\
\hline Catalogación Descriptiva & 6 \\
\hline Lenguajes Documentales I & 6 \\
\hline Archivistica I & 6 \\
\hline Edición Digital & 6 \\
\hline Inglés Documental I & 6 \\
\hline
\end{tabular}

Tercer curso

\begin{tabular}{|l|c|}
\hline Fondos Bibliográficos Antiguos & 6 créditos \\
\hline $\begin{array}{l}\text { Planificación, Diseño y Evaluación de Unidades de } \\
\text { Información y Documentación }\end{array}$ & 6 \\
\hline Catalogación Automatizada & 6 \\
\hline Lenguajes Documentales II & 6 \\
\hline Búsqueda y Recuperación de Información & 6 \\
\hline Archivística II & 6 \\
\hline Fundamentos y Diseño de Bases de Datos & 6 \\
\hline $\begin{array}{l}\text { Sistemas Automatizados en Unidades de Infor- } \\
\text { mación }\end{array}$ & 6 \\
\hline Dos optativas & 12 \\
\hline
\end{tabular}




\begin{tabular}{|l|c|}
\hline \multicolumn{2}{|c|}{ Cuarto curso } \\
\hline $\begin{array}{l}\text { Administración y Gestión de Unidades de Infor- } \\
\text { mación y Documentación }\end{array}$ & 6 créditos \\
\hline Bibliometría & 6 \\
\hline Metodología de la Investigación & 6 \\
\hline Prácticas Externas & 6 \\
\hline Trabajo de Fin de Grado & 6 \\
\hline Cinco optativas & 30 \\
\hline
\end{tabular}

\begin{tabular}{|l|c|}
\hline \multicolumn{2}{|c|}{ Optativas de tercer curso } \\
\hline Documentación Musical & 6 créditos \\
\hline $\begin{array}{l}\text { Documentación de la Unión Europea y Publicacio- } \\
\text { nes Oficiales }\end{array}$ & 6 \\
\hline $\begin{array}{l}\text { Fuentes de Información y Gestión Documental } \\
\text { para las Administraciones Públicas }\end{array}$ & 6 \\
\hline Análisis y Lenguajes Documentales Especiales & 6 \\
\hline Estudio del Documento Medieval & 6 \\
\hline $\begin{array}{l}\text { Estudio del Documento Moderno y Contemporá- } \\
\text { neo }\end{array}$ & 6 \\
\hline Fuentes Archivísticas & 6 \\
\hline $\begin{array}{l}\text { Digitalización, Bibliotecas Digitales y Repositorios } \\
\text { Documentales }\end{array}$ & 6 \\
\hline Inglés Documental II & 6 \\
\hline Latín Práctico para Archivos y Bibliotecas & 6 \\
\hline
\end{tabular}

\begin{tabular}{|l|c|}
\hline \multicolumn{2}{|c|}{ Optativas de cuarto curso } \\
\hline Políticas de Información y Documentación & 6 créditos \\
\hline $\begin{array}{l}\text { Derechos Humanos, Ciudadanía y Sociedad de la } \\
\text { Información }\end{array}$ & 6 \\
\hline Marketing en Internet & 6 \\
\hline Documentación Fotográfica & 6 \\
\hline Estudios y Formación de Usuarios & 6 \\
\hline Sistemas de Recuperación e Internet & 6 \\
\hline Archivos Electrónicos & 6 \\
\hline Redes y Seguridad & 6 \\
\hline Introducción a la Programación & 6 \\
\hline $\begin{array}{l}\text { Derecho de la Documentación y su Régimen Ju- } \\
\text { rídico }\end{array}$ & 6 \\
\hline $\begin{array}{l}\text { Historia del Derecho y de las Instituciones Espa- } \\
\text { ñolas (Siglos XVIII-XXI) }\end{array}$ & 6 \\
\hline
\end{tabular}

Créditos de participación (cualquier curso) 


\section{Universidad de Sheffield ${ }^{10}$}

Al igual que en el resto de las universidades británicas los programas de estudio de la Universidad de Sheffield presentan una estructura modular, es decir, basada en créditos, lo que le permite al estudiante una cierta flexibilidad a la hora de diseñar su propio curso académico. Actualmente la Universidad de Sheffield ofrece 5 Titulaciones en su Facultad de Información que tienen relación con el ámbito de la Información, la Documentación, los Negocios y la Gestión de la Información (todas tienen una duración de 3 cursos).

- BA in Accounting Financial Management and Information Management.

- BA in Business Management and Information Management.

- BSC in Information Management.

- BSC in Information Management for Business.

- BSC in Information Management and Technology.

Únicamente se ha hecho un análisis y un conjunto de propuestas de mejora para las asignaturas de los planes de estudio de las tres primeras titulaciones, ya que las otras dos no aparecen reflejadas en la página Web de la Facultad. En este sentido las asignaturas comunes a los planes de estudio de $B A$ in Accounting Financial Management and Information Management, BA in Business Management and Information Management y BSC in Information Management son las siguientes:

- Introduction to Information Management.

- Information Management in the Learning Organization.

- Communication Networks in Organizations.

- Information Storage and Retrieval.

- Information Management Project.

- Information Literacy.

De esta forma se establece la posibilidad de obtener las tres titulaciones en un tiempo mucho más reducido que si se cursasen por separado, lo que aumenta las posibilidades de empleo de los alumnos. Además se cuenta con la gran tradición docente del Departamento de Biblioteconomía y Documentación de la Facultad de Información de la Universidad de Sheffield, cuya fundación se remonta a 1963. 
Del análisis del plan de estudios de la Universidad Complutense de Madrid se pueden extraer las siguientes conclusiones:

- El plan de estudios del Grado en Información y Documentación de la Universidad Complutense de Madrid prepara al futuro graduado para trabajar en las siguientes áreas:

- Como Documentalista en las Administraciones Públicas en todos sus niveles.

- En Archivos de Gestión de Municipios, Consejerías, Ministerios, etcétera.

- En empresas privadas: medios de comunicación, empresa editorial, empresas de contenidos multimedia, etcétera.

- Este plan de estudios está muy centrado en cuatro áreas:

- Biblioteconomía.

- Documentación.

- Archivística.

- Tecnologías de la Información y de la Comunicación (TIC).

- Faltan créditos en asignaturas relativas a la organización y gestión de los archivos, las bibliotecas y los centros de documentación.

- Además son escasos los créditos de asignaturas relativas a otras áreas de conocimiento (Periodismo, Historia, Economía, Derecho).

- Las optativas no están organizadas por itinerarios.

- Faltan más asignaturas de lenguas clásicas (Latín, Griego).

- Faltan más asignaturas de lenguas modernas (Inglés, Francés, Alemán, Italiano).

- Los 6 CR. ECTS de prácticas externas son insuficientes.

- Los 6 CR. ECTS del Trabajo de Fin de Grado son insuficientes.

Por tanto del plan de estudios del Grado en Información y Documentación de la Universidad Complutense de Madrid se deduce que está orientado a la formación de especialistas en Archivística, Biblioteconomía y Documentación, pero esto no está suficientemente valorado ni requerido en los procesos selectivos de las oposiciones a la Administración Pública. En primer lugar porque se exige cualquier titulación superior universitaria para optar a la oposición, lo que reduce las oportunidades de conseguir una plaza pública por parte de los graduados en Información y Documentación al tener que competir con Licenciados en otras disciplinas.

Del análisis del plan de estudios de la Universidad de Sheffield se pueden extraer las siguientes conclusiones: 


\section{Generales}

- Las diplomaturas de BA in Accounting Financial Management and Information Management, BA in Business Management and Information Management y BSC in Information Management tienen una duración de tres años, periodo de tiempo en el cual los universitarios deben de aprobar un total de 360 créditos, 120 por año lectivo. Las asignaturas se clasifican por Niveles, que hacen referencia a la dificultad de sus contenidos, de esta forma, las que corresponden al Nivel 1 pertenecen al primer año de carrera y las del Nivel 3 al último año. El alumno, dependiendo del curso académico en que se encuentre, podrá matricularse en aquellas asignaturas que, perteneciendo a su Nivel correspondiente, considere más interesantes.

- Estos planes de estudios están muy enfocados a la gestión de la información, y a la organización y gestión de la documentación empresarial.

- Por tanto los estudiantes serán capaces de aportar soluciones integrales, flexibles y fiables a la gestión de la información. El objetivo primordial es saber diseñar un sistema de gestión de recursos de información aplicable a cualquier tipología empresarial, así como conocer las herramientas informáticas de vanguardia para la organización, gestión y recuperación de la documentación.

- Son inexistentes las materias relativas a las áreas de Archivística, Biblioteconomía y Documentación.

- La mayoría de las asignaturas son comunes a las tres titulaciones. En este sentido, se permite la transversalidad entre ellos y la posibilidad de obtener las tres titulaciones en un tiempo mucho más reducido que si se cursasen por separado. Esto aumenta las posibilidades de empleo de los alumnos al tener una formación muy versátil.

- Este plan de estudios posibilita que el alumno configure su propio currículum académico en función de sus intereses y preferencias, ya que tiene una elevada carga lectiva de optativas.

- Faltan asignaturas de lenguas extranjeras (Español, Francés, Alemán).

\section{Especificas}

- Los planes de estudio constan de una gran variedad de asignaturas:

- Relativas a las Redes de Comunicación Empresarial (Information and Communication Networks in Organizations). 
- Relativas a la Gestión de la Información (Introduction to Information Management).

- Relativas a los Modelos de Sistemas de Información (Information Systems Modelling).

- Relativas al Diseño de Bases de Datos (Database Design).

- Relativas a los Sistemas de Información y la Sociedad de la Información (Information Systems and Information Society).

- Relativas a la Gestión y Estrategias de Información (Information Management and Strategy).

- Además, se introducen algunas asignaturas complementarias como Marketing (Principles of Marketing) y Economía Financiera (Financial Accounting).

- También se cursan asignaturas muy novedosas:

- Alfabetización de la Información (Information Literacy).

- Introducción a la Investigación de la Información (Introduction to Information Research).

- Introducción al Comportamiento en el Trabajo (Introduction to Behaviour at Work).

- Introducción a la Investigación en la Información (Introduction to Information Research).

- Recuperación de Documentos: Motores de Búsqueda y Bibliotecas Digitales (Information Retrieval: Search Engines and Digital Libraries).

- El tercer curso tiene menos asignaturas con el objetivo de que los alumnos se especialicen en aquellas áreas que sean de su interés.

Por tanto, del plan de estudios de la Universidad de Sheffield se deduce que educa profesionales especializados en Documentación Empresarial sustentados en las Tecnologías de la Información y de la Comunicación (TIC), en detrimento de los conocimientos más propios del ámbito de la información y de la documentación, como la catalogación informatizada, la utilización de la Clasificación Decimal Universal (CDU), los estudios bibliométricos y la evaluación de sistemas y usuarios de información y documentación.

\section{MEJORAS EN LOS PLANES DE ESTUDIOS}

En cuanto a las mejoras correspondientes al Plan de Estudios del Grado en Información y Documentación de la Universidad Complutense de Madrid serían deseables las siguientes: 
- Aumentar la carga lectiva en asignaturas relacionadas con la gestión de los archivos, las bibliotecas y los centros de documentación.

- Implantar asignaturas enfocadas a la gestión de la Documentación Empresarial.

- Introducir asignaturas relativas a otras áreas de conocimiento y optativas especializadas con el objetivo de ampliar la formación de los futuros graduados, por la iniciativa ya consolidada en algunas Universidades europeas de recuperar las materias humanísticas para la formación integral de la persona. Por ejemplo, Geografía de España, Historia del Arte Español, Patrimonio Histórico Artístico Español, Derecho de la Información, Museología, Economía Financiera, Fuentes de Información Jurídica, Fuentes de Información en Economía y Empresa, Alfabetización de la Información, etcétera.

- Organizar las asignaturas optativas por itinerarios: Documentación Especializada, Biblioteconomía, Archivística, Documentación Jurídica y Documentación Empresarial con vistas a que los graduados tengan mejores salidas profesionales.

- Aumentar la carga lectiva en lenguas clásicas (Latín, Griego) para fomentar la capacidad de desarrollar un pensamiento crítico por parte de estos profesionales.

- Introducir asignaturas de lenguas modernas (Inglés, Francés, Alemán) para que los titulados desarrollen su actividad profesional en un ámbito internacional.

- Establecer 18 créditos ECTS de prácticas externas para que los graduados puedan tener más experiencia profesional antes de finalizar sus estudios.

- Asignar una carga lectiva de 12 créditos ECTs al Trabajo de Fin de Grado para que los estudiantes puedan investigar y les sirva como paso previo si desean cursar un Máster Oficial y un Doctorado.

- Introducir en el plan de estudios asignaturas relativas a técnicas de trabajo en grupo, oratoria y habilidades comunicativas para potenciar las perspectivas profesionales de los graduados, ya que, según investigaciones recientes, las empresas demandan estas habilidades de las que carecen la mayoría de los programas académicos de las Universidades de España.

En lo concerniente a las mejoras correspondientes al Plan de Estudios del Grado en Información y Documentación de la Universidad de Sheffield serían deseables las siguientes: 
- Aumentar la carga lectiva en asignaturas relacionadas con la Biblioteconomía (Historia del Libro y de las Bibliotecas, Bibliometría, Bibliografía y Fuentes de Información).

- Ampliar la oferta formativa en asignaturas relacionadas con la Documentación (Lenguaje Documental, Documentación General, Análisis Documental).

- Incorporar materias relacionadas con la Archivística (Instituciones Modernas y Contemporáneas y su Producción Documental, Organización de Archivos, Sistemas Archivisticos).

- Introducir asignaturas de lenguas clásicas (Latín, Griego) para promover una formación humanística de los graduados.

- Implantar seminarios especializados con la finalidad de que los graduados adquieran las técnicas documentales necesarias para trabajar en bibliotecas, archivos y centros de documentación especializados.

- Introducir asignaturas de lenguas modernas (Alemán, Español, Francés) con el objetivo de que, al encontrarnos en una sociedad globalizada y multicultural, los futuros profesionales puedan desempeñar su trabajo en cualquier lugar del mundo.

Esta propuesta de reforma sobre los mencionados planes de estudio también está refrendada por un informe elaborado por la Fundación Banco Bilbao Vizcaya Argentaria (BBVA) (2011), en el que la mayoría de los jóvenes europeos que cursan estudios superiores cree que existe un importante desajuste entre la formación que reciben y la vida laboral. El estudio se ha realizado con estudiantes universitarios de seis países europeos (Reino Unido, Francia, Suecia, Alemania, Italia y España), basado en una encuesta a 18.000 jóvenes, 3.000 por cada Estado.

En este sentido cabe destacar que la valoración de la preparación teórica y profesional ofrecida por las Universidades es muy diferente entre los jóvenes de los países encuestados. De hecho la opinión de la mayoría de los británicos es claramente positiva, ya que el 70\% piensa que la Universidad prepara adecuadamente a los estudiantes para su futura vida profesional. Lo mismo considera el 50\% de los estudiantes suecos y alemanes. Sin embargo la opinión de los jóvenes españoles, italianos y franceses es mucho más desfavorable. En España, el 54\% expresa que existe un claro desajuste entre formación y mercado laboral, frente al $40 \%$ que sostiene lo contrario. Por lo que respecta a la rama de estudio, se observa que en casi todos los países los estudiantes de Ciencias de la Salud y de carreras técnicas tienden a valorar más favorablemente la preparación profesional, mientras que los de Ciencias Sociales y Humanidades acusan en mayor medida las carencias en este tipo de formación. 
Por estas razones las mejoras planteadas tienen como objetivo primordial que los futuros graduados alcancen el éxito profesional en consonancia con la formación académica recibida en la Universidad. De esta forma, a través de una metodología práctica, se consigue la adquisición de las competencias profesionales necesarias recogidas en los planes de estudio adaptados al Espacio Europeo de Educación Superior (EEES), vigente en 47 países.

\section{Conclusiones}

Las actividades básicas del profesional de la información y la documentación son las relacionadas con localizar la información, analizarla y tratarla para aumentar su valor para quien la tiene que utilizar, y gestionarla, convertirla en fácilmente accesible y transmitirla a los usuarios o clientes que la necesitan. En definitiva, contribuyen a ahorrar tiempo en las organizaciones. Para ello tienen que adquirir competencias relacionadas con el manejo de las tecnologías, la comunicación y la gestión, así como las propias de aquellos campos de actividad en la que desarrollan su trabajo.

En el ámbito público, en la mayoría de las convocatorias oficiales de oposiciones, no es frecuente que sea solicitada la titulación de Grado en Información y Documentación, Licenciado en Documentación o Diplomado en Biblioteconomía y Documentación. Esto da lugar a situaciones ilógicas, como por ejemplo que los Licenciados en cualquier titulación ajena a Biblioteconomía puedan aprobar las Oposiciones a Ayudante de Biblioteca del Ministerio de Cultura. Sin embargo en las convocatorias oficiales de Becas de Formación para Postgraduados (Senado, Consejo Superior de Deportes, Instituto Cervantes, Comunidad de Madrid) sí es solicitada expresamente esta titulación.

En el ámbito privado, en la mayoría de los casos, tampoco se suele solicitar formación universitaria en Biblioteconomía y Documentación (excepción hecha de las Universidades Privadas -como la Europea de Madrid-, Repsol y las Bibliotecas de Fundaciones). Sin embargo, en cuanto a conocimientos específicos del ámbito de la documentación, sí se ha constatado la exigencia de conocer Sistemas de Gestión de Bibliotecas como UNICORN, ABSYS y MARC21.

En cuanto al ámbito académico, al comparar las dos Universidades investigadas que han sido seleccionadas por su larga trayectoria en estos estudios, se infiere que el Grado en Información y Documentación de la Universidad Complutense de Madrid se estructura en cuatro cursos, mientras que los de la Universidad de Sheffield lo hacen en tres. Además, el plan de estudios de 
la Universidad Complutense de Madrid está muy focalizado en las áreas de Biblioteconomía, Documentación, Archivística y Tecnologías de la Información, mientras que los de la Universidad de Sheffield se centran en la gestión de la información, la organización y gestión de la documentación empresarial y el diseño de sistemas de gestión de recursos de información.

Así, en el plan de estudios de la Universidad Complutense es necesaria una mayor oferta de asignaturas del ámbito empresarial y humanístico y una sólida formación en idiomas clásicos y extranjeros. Por su parte en el programa de la Universidad de Sheffield sería conveniente reforzar las áreas de Biblioteconomía, Archivística y Documentación, así como las lenguas clásicas y modernas.

En el Grado de Información y Documentación sería conveniente realizar algunas reformas de contenido en los planes de estudios que puedan situar a sus graduados en la vanguardia del conocimiento. Para paliar estas deficiencias que afectan a los futuros profesionales sería deseable, al igual que lo tienen los abogados y los médicos, la creación del Colegio Oficial de Archiveros, Bibliotecarios y Documentalistas en el ámbito nacional, lo que redundaría en un mayor reconocimiento social y profesional de sus titulados. Consecuentemente, mejoraría el perfil del profesional que solicitan los empleadores, tanto en el ámbito público como en el privado.

Además, para sucesivas investigaciones concernientes a saber si existe relación o falta de vinculación entre el futuro profesional que se forma en la Universidad y lo que demanda el mercado, sería muy pertinente aunar los contenidos de los programas académicos de estas dos prestigiosas Universidades (Complutense de Madrid y Sheffield). De esta forma, se conseguiría una correspondencia real entre formación académica y demanda del ámbito laboral para formar profesionales competentes y versátiles en el área de Información y Documentación.

\section{BiBLIOGRAFÍA}

Alonso Arévalo, J., Vázquez Vázquez, M. (2000), “Características del comportamiento del mercado de trabajo en Biblioteconomía, Archivística y Documentación, bienio 98/99", en Anales de documentación, vol. 3, pp. 9-24.

ANECA (Agencia Nacional de Evaluación de la Calidad y la Acreditación), Libro Blanco del Título de Grado en Información y Documentación, (2004), http://www.aneca.es/var/media/150424/libroblanco_jun05_documentacion.pdf [consultado: 25 de julio de 2011]. 
Artaza, C. H. (2000), "El mercado laboral de los titulados en Bibliotecología y Documentación: análisis de las ofertas de empleo publicadas en Argentina", en Investigación Bibliotecológica, vol. 25, núm. 54, pp. 17-36.

Azevedo L. J., Gomez, S. (2006), "O mercado de trabalho para os profissionais da informacao no contexto de empresas brasileiras das regiones geográficas norte, nordeste e centro-oeste", en Informação \& Sociedade 16(1), disponible en: ttp://www.ies.ufpb.br/ ojs2/index.php/ies/article/view/457/1508 [consultado: 24 de enero de 2009].

Comalat Navarra, M.; Espelt Busquets, C. y Rubió Rodón, A. (2003), "Nuevos yacimientos laborales para diplomados: análisis de los convenios de cooperación educativa de la Facultad de Biblioteconomía y Documentación”, (UB), 1998-2002, en Revista de Biblioteconomía i Documentació, vol. 33, 71-78.

Diario Oficial de la Comunidad Europea (DOCE), http://eur-lex.euro pa.eu/JOIndex.do?ihmlang=es [consultado: 29/07/2011].

Frías Montoya, J. A. et al (2000), "Formación e inserción laboral de los titulados universitarios en Biblioteconomía y Documentación por la Universidad de Salamanca”, en $V$ Encuentro de la Asociación de Educación e Investigación en Bibliotecología, Archivología, Ciencias de la Información y Documentación de Iberoamérica y El Caribe, pp. 475-492, Granada, España.

INFODOC, http://listas.bcl.jcyl.es:81/read/messages?id=9508\#9508 [consultado: 15 de julio de 2011].

Montes López, E. (1995), "El mercado de trabajo de los documentalistas en España: análisis de una muestra de ofertas de empleo, 1984-1994", en Revista Española de Documentación Científica, vol.18, (2), pp. 178-187.

Moreiro González, J. A. (2001a), "Figures on employability of Spanish library and information science graduates", Libri, 2001, vol. 51 (1), pp. 27-37.

(2001b), "Licenciatura en documentación: experiencias, desarrollo y problemática, El caso de la Universidad Carlos III de Madrid", en Anales de Documentación, vol. 4, pp. 151-168.

Muñoz-Cañavate, A. et al., "Un estudio sobre las nuevas posibilidades de trabajo para los titulados en Biblioteconomía y Documentación: la gestión de información corporativa en las Administraciones Públicas en la red", en Jornadas Españolas de Documentación (10. ${ }^{a}$ 2007. Santiago de Compostela), E- información: integración y rentabilidad en el entorno digital (2007), pp. 373-382.

Pineda J. M. (2002), "Realidad laboral de los bibliotecarios y bibliotecólogos egresados de la Escuela de Bibliotecología, dependiente de la Facultad de Filosofía, de la Universidad Nacional de Córdoba (Argentina), en Revista Biblios, Núm 12, abril - junio, pp. 1-23, disponible en: http://dialnet.unirioja.es/servlet/fichero_articulo? codigo $=254958$ \&orden $=0$ [consultado: 20 de marzo de 2009]. 
Ríos Hilario, A. B. y Sánchez Santos, M., (2011), "El perfil actual del personal catalogador: adecuación entre la formación universitaria y la demanda laboral", en Anales de Documentación, vol. 14, núm. 2, en http://revistas.um.es/analesdoc/article/view/131461.

Tejada Artigas, Carlos y Rodríguez Yunta, Luis, (2007), "Un acercamiento a la situación laboral y desarrollo profesional de los trabajadores de la información y documentación: la encuesta SEDIC 2006”, en Jornadas Españolas de Documentación (10. a 2007. Santiago de Compostela) E-información: integración y rentabilidad en el entorno digital, pp. 383-392.

Ubieto Artur, María Isabel, et al., (2007), "Evolución de la oferta de empleo público en Biblioteconomía y Documentación en España durante la década 1996-2006: avance de una investigación”, en Jornadas Españolas de Documentación (10. a 2007, Santiago de Compostela) E-información: integración y rentabilidad en el entorno digital, pp. 393-401.

Universidad Complutense de Madrid, Facultad de Ciencias de la Documentación http://www.ucm.es/?a=estudios $\& \mathrm{~d}=$ muestragrado 3 \&idgr=23 [consultado: 18 de julio de 2011].

Universidad Complutense de Madrid. Consejo Social (2001), Trayectorias laborales de los diplomados en Biblioteconomía y Documentación, Madrid, pp. 65-67.

Universidad de Barcelona. Facultat de Biblioteconomia i Documentació (2003), Diplomatura en Biblioteconomia i Documentació, informe d'autoavaluació, versió definitiva que incorpora els comentaris rebuts de la comunitat universitaria, Barcelona, Universitat de Barcelona, Facultat de Biblioteconomia i Documentació, pp. 18-19.

Universidad de Sheffield, http:/www.shef.ac.uk/is/study/ug/courses [consultado: 27 de julio de 2011].

Universidad Politécnica de Madrid, Ofertas de Empleo Público. http://www.upm.es/institucional/PAS/PASFuncionario/Concursos yOposiciones/AccesoLibre/SubgrupoA1/0bf18278f45d9210VgnV CM10000009c7648aRCRD [consultado: 12 de julio de 2011]. 
